\title{
Experimental Study on the Influence of Pulse Load on Micro - grid Operating Characteristics
}

\author{
Chuang LV*, Pu XIE, Zhi-Yong YIN \\ Department of Vehicle and Electrical Engineering, Ordnance Engineering College, Shijiazhuang \\ 050003, China \\ *Corresponding author
}

\section{Keywords: Pulse Load, Microgrid, Voltage Distortion Rate, Experimental Study.}

\begin{abstract}
The structure of Pulse load is complicated and the load characteristics is closely related to the work mode, in order to study the micro grid operation characteristics under pulse load and the different parameters of pulse load on the influence of the micro grid operation characteristics. This paper built a test system of diesel generator sets and pulse load and established the pulse load simulator, and defined the calculation formula of the voltage fluctuation under the premise of considering the system frequency fluctuation. Analyzed the influence law on voltage and frequency fluctuation, power transmission efficiency and the voltage distortion degree in different working mode of pulse load through changed the duty ratio, working cycle and peak power. Results show that the micro grid operation characteristics has a great relationship with the operation mode of pulse load, and different pulse load parameters on the micro grid operation characteristics of the influence law and degree is different.
\end{abstract}

\section{Introduction}

Diesel generator is an important form of mobile equipment power supply, can be used as an important backup power supply, but also an important power supply micro-power grid. With a small size, start quickly, reliable power supply, easy maintenance features. In the practical application has been widely used. The pulse period is usually tens or hundreds of milliseconds, the power consumption is not stable, showing a typical pulse characteristics, with a low average power, Peak power of the characteristics of large. Different from the single load at start and stop, the pulse load not only has an impact on the power supply at start-up and shutdown, but also in the normal operation, the power line is repeatedly loaded and unloaded due to the power mutation. Of the change, thus affecting the power output characteristics.

In the study of microgrid system with pulse load, the working mechanism of pulse load is studied in [1], and three kinds of pulse load structures which can be used for experimental analysis are designed, which lays the foundation for carrying out pulse load test and theoretical research. [2] An integrated power system with pulse load is established, and the influencing factors of system steady state are analyzed by simulation. In the literature [3], a single small-capacity generator in the ship's power system is used as the research background. The system voltage and current are analyzed by modeling and simulation. [4] The active control of the microgrid under pulsed load is studied. In view of the overcurrent phenomenon that may occur in the diesel power supply system, the method of improving the stability of the system through the reasonable energy storage control is put forward. The influence of the pulse load on the AC voltage and frequency is analyzed in the literature [5]. However, the above documents are based on the establishment of pulse load simulation model to analyze the pulse load on the impact of diesel generating units, not taking into account the actual output characteristics of diesel generating units by the running time of the heat a greater impact, and the governor and excitation The output voltage of the voltage regulator and the output voltage of the controllable rectifier are affected by the actual operating conditions, and there is a certain deviation from the simulation results under the ideal conditions. The above documents are not related to the pulse load in different operating modes, that is, the parameters of the pulse load Ratio D, duty cycle TS, peak power PL) changes on the operating characteristics of diesel generators for a detailed study of the 
law ${ }^{[6]}$. In order to study the effect of pulse load on the operating characteristics of microgrid, this paper takes the diesel generator - pulse load test system as the research object, according to the characteristics of pulse load to establish a pulse load simulation device, and according to different micro-grid operating parameters given The corresponding definition formula, focusing on the pulse load in different modes of operation on the microgrid operating characteristics of the law of a detailed study[7].

\section{Experimental System Structure and Principle}

The test system consists of a single diesel generator and pulse power load simulation device. System equivalent circuit shown in Fig. 1, where the electronic governor in accordance with the system in real time to adjust the diesel engine power to maintain the unit output frequency stability, automatic voltage regulator (AVR) control diesel generator output voltage stability, controllable rectifier adjustment The system DC side of the output voltage is maintained at the set value of $500 \mathrm{~V}$, the system uses LC passive filter, DC switch off by the external trigger signal control, pulse pulse can be set to adjust the pulse load mode of operation. In this power supply system, the diesel generator set as a DC power supply to the pulse load. Pulse load simulation device consists of rectifier cabinet, DC switchgear, DC load cabinet. The control panel of the rectifier cabinet can set the DC output voltage, open loop or closed loop mode, the display can display the AC side voltage, current, power average and DC side voltage, current real time value; DC switchgear touch screen can be set to DC switch on and off, the duty cycle TS, duty cycle D, the display can display the DC voltage, current peak; DC load cabinet control panel can be set cabinet alarm temperature, Load the number of groups, each load $10 \mathrm{~kW}$, a total of 10 groups, you can set the pulse load peak power between $10 \mathrm{~kW}-100 \mathrm{~kW}$, the display can display the real-time cabinet temperature, load power. In the test system, the rated power of the diesel engine is $50 \mathrm{kw}$, the rated speed is $1500 \mathrm{r} / \mathrm{min}$, the electronic speed control; synchronous generator is a three-phase brushless synchronous generator with a pole pair number of 2 , rated capacity of $72.5 \mathrm{kVA}$, power factor of 0.8 , Using MX321 type excitation voltage regulator. The rated output line voltage of the unit is $400 \mathrm{~V}$ and the output frequency is $50 \mathrm{~Hz}$. Pulse load flat-wave reactance $\mathrm{L}$ is $0.125 \mathrm{mH}$, filter capacitor $\mathrm{C}$ is $4000 \mu \mathrm{F}$, the experimental data collected by the DL850 oscilloscope, sampling frequency of $20000 \mathrm{~Hz}$.

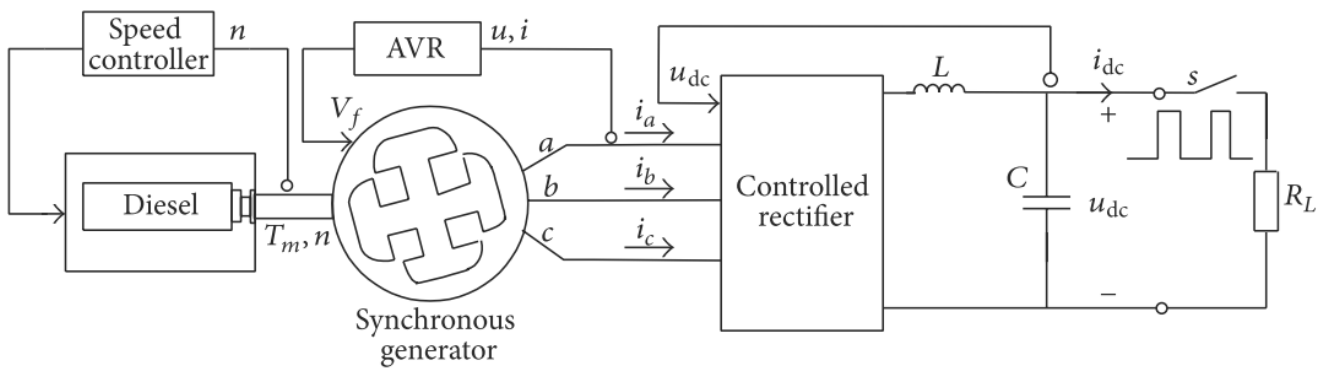

Fig. 1.diesel generator -pulse load equivalent circuit diagram 


\section{Test Results and Analysis}

\section{Simulation Results and Analysis}

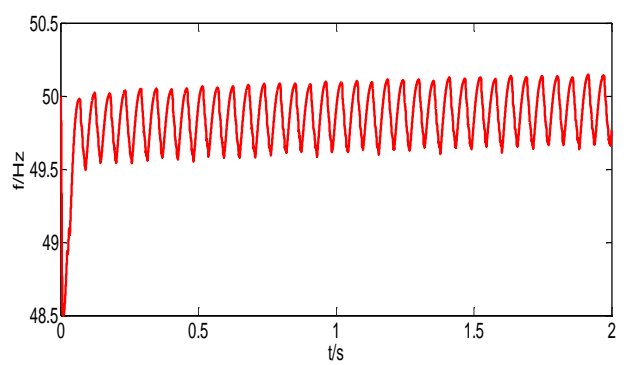

a Output frequency waveform of diesel generator set

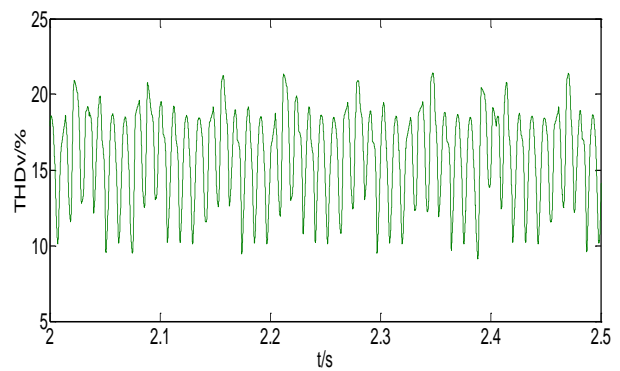

c Diesel engine voltage distortion rate

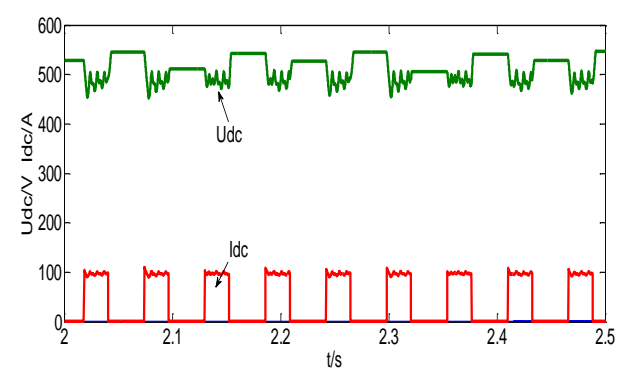

b DC side load voltage and current waveform

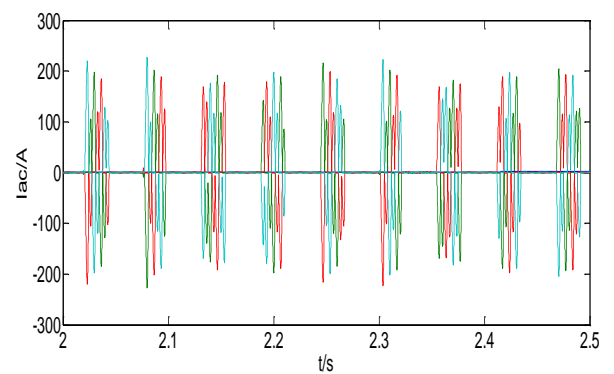

d Diesel generator output three - phase current waveform

Fig. 2. pulse load in a specific mode when the electrical quantity waveform

Fig. 2 shows the peak power of $50 \mathrm{~kW}$, the output duty cycle of 0.4 , the duty cycle of $56 \mathrm{~ms}$ pulse load filter capacitor $4000 \mathrm{uF}$ when the voltage and current, frequency, voltage distortion rate of the waveform, the waveform can be seen, the current Continuous pulse-like, when the line current output, the DC voltage decreases, and when there is no output current in the line, the DC voltage increases; And because of the periodic fluctuation of the pulse load, the frequency of the diesel generator will fluctuate slightly in the vicinity of a certain fixed value. It can be seen from Figure $c$ that the voltage distortion during the pulse load is very serious and the distortion amplitude fluctuates Big. And the distortion of the voltage will have a certain impact on the efficiency of diesel engine power transmission.

In order to further study the influence of pulse load on the voltage fluctuation, frequency fluctuation, power transmission efficiency and voltage distortion rate of the microgrid, the duty cycle, duty cycle and peak power of the pulse load were changed to analyze the influence of the pulse load parameters on the above evaluation index.

\section{Influence of Duty Cycle on Dynamic Characteristics of System}

Table 1 System dynamics when changing the duty cycle (P50_T56)

\begin{tabular}{cccccc}
\hline $\mathrm{D}$ & $\Delta \mathrm{f} / \%$ & $\mathrm{Udc} / \mathrm{V}$ & $\delta_{\mathrm{u}} / \%$ & $\mathrm{Pdc} / \mathrm{KW}$ & $\eta / \%$ \\
\hline 0.1 & 1.31 & 501.8 & 10.32 & 4.50 & 90.1 \\
0.3 & 2.91 & 491.5 & 16.93 & 11.85 & 79.6 \\
0.5 & 5.21 & 499.2 & 20.38 & 21.03 & 84.5 \\
0.7 & 3.57 & 486.7 & 15.38 & 30.83 & 88.4 \\
0.8 & 1.79 & 487.1 & 12.51 & 36.04 & 91.2 \\
1.0 & 0.21 & 495.6 & 1.37 & 45.93 & 92.1 \\
\hline
\end{tabular}

Table 1 to change the duty cycle when the dynamic characteristics of the system can be seen from Table 1, only to change the duty cycle, the diesel generator output frequency and DC voltage average 
in the vicinity of the rated value of small fluctuations and pulse load power consumption As the duty cycle increases linearly. And the change of duty cycle has little effect on the power transmission efficiency. When the duty cycle is small (about less than 0.5 ), the voltage fluctuation increases with the increase of duty cycle, when the system load increases, the load power increases with the duty cycle increases, the diesel generator When the duty cycle is larger (about greater than 0.5), the voltage fluctuation decreases with the increase of the duty ratio. At this time, as the duty ratio increases, the pulse load average power Gradually increases, the average load power is close to the power capacity, due to increased load current caused by the impact of the pulse load on the unit to reduce the impact.

\section{The Impact of The Duty Cycle on The Dynamic Characteristics of The System}

Table 2. System dynamics when changing the duty cycle (P50_D40)

\begin{tabular}{llllll}
\hline $\mathrm{Ts} / \mathrm{ms}$ & $\Delta \mathrm{f} / \%$ & $\mathrm{Udc} / \mathrm{V}$ & $\delta_{\mathrm{u}} / \%$ & $\mathrm{Pdc} / \mathrm{kw}$ & $\eta / \%$ \\
\hline 10 & 0.53 & 497.4 & 9.73 & 17.11 & 85.5 \\
30 & 1.84 & 490.7 & 13.35 & 17.42 & 87.1 \\
56 & 3.58 & 493.7 & 18.54 & 17.92 & 89.6 \\
80 & 6.31 & 478.2 & 22.34 & 18.45 & 92.3 \\
100 & 6.58 & 497.5 & 24.59 & 18.33 & 91.7 \\
\hline
\end{tabular}

Table3.Systemdynamics when changing peak power (D40-T56)

\begin{tabular}{clllll}
\hline $\mathrm{P}_{\mathrm{l}} / \mathrm{kw}$ & $\Delta \mathrm{f} / \%$ & $\mathrm{Udc} / \mathrm{V}$ & $\delta_{\mathrm{u}} / \%$ & $\mathrm{Pdc} / \mathrm{kw}$ & $\eta / \%$ \\
\hline 10 & 1.28 & 502.5 & 4.92 & 3.66 & 91.46 \\
30 & 3.01 & 503.1 & 13.25 & 9.99 & 83.24 \\
50 & 4.68 & 493.7 & 18.54 & 16.27 & 81.35 \\
70 & 6.34 & 482.4 & 22.53 & 19.45 & 69.57 \\
80 & 7.13 & 483.3 & 23.48 & 19.84 & 61.87
\end{tabular}

Table 2 shows the dynamic characteristics of the system when the duty cycle is changed. As can be seen from Table 2, with the increase of duty cycle, the output frequency of diesel generator is not changed as a whole, but the frequency fluctuation is very significant, It can be seen from the table that the frequency fluctuation rate increases with the increase of the duty cycle of the load, because the increase of the voltage fluctuation increases with the increase of the pulse load working time, which leads to the increase of the frequency fluctuation. In the case of constant duty cycle, increasing the duty cycle of the pulse load is equivalent to increase the working time of the pulse load, thus the greater impact on the unit excitation voltage regulator, and the increase in the cycle will lead to the number of cycles per unit time Reduce, so from the table can be seen that the pulse power consumption of the average power change is not. And the increase of the working time due to the increase of the working time leads to the increase of the peak power, which requires the capacitor to supply more power. Therefore, the time of the charge of the pulse-gap generator is correspondingly longer, which is equivalent to that of the AC-side high-power load. The voltage fluctuation rate increases with the increase of the duty cycle.

\section{Influence of Peak Power on Dynamic Characteristics of System}

Table 3 shows the dynamic characteristics of the system when the peak power is changed. It can be seen from Table 3 that changing only the peak load of the pulse load is equivalent to changing the average power of the pulse load, so the change is the same as changing the duty cycle, The load power consumption and the frequency fluctuation rate increase linearly with the peak load of the 
pulse load. When the peak power is changed, the output frequency and the DC voltage of the diesel engine are basically stable, and the voltage fluctuation is very significant. The larger the peak power, the greater the voltage fluctuation is because the actual power of the load increases when the peak power increases Large, resulting in a more intense pulse load in the system, so the diesel generator output voltage by the load current changes greater impact.

\section{Conclusion}

In the diesel generator - pulsed load test system, by comparing the influence of pulse load on the running characteristics of the system under different working modes, the following conclusions are obtained:

1) pulse load operation will produce DC voltage fluctuations and AC frequency fluctuations, and its changes with the work cycle, the peak power showed a monotonically increasing relationship between the change, and the relationship between the duty cycle is more complex, when the duty cycle is about 0.5 when the voltage And the frequency of the most serious fluctuations;

2) micro-grid power transmission efficiency and pulse load duty cycle,The relationship between the work cycle is not great, the correlation with the peak power is the largest, and the higher the peak power, the lower the power transmission efficiency;

3) The voltage distortion of microgrid is related to duty cycle, duty cycle and peak power, and the voltage distortion rate is positively correlated with duty cycle and peak power, and negatively correlated with working cycle.

\section{Acknowledgments}

This work was financially supported by National Natural Science Foundation of China (No. 51307184) and Science and technology project of Hebei Province(No.15210701D).

\section{References}

[1] LIU Zhengchun, WANG Yong, YIN Zhiyong, et al. Modeling and simulation of pulsed power load inlimited capacity system[J]. Journal of North China Electric Power University, 2014, 41(1): 33-37.

[2] Wang Yong, Liu Jin-ning, Liu Zheng-chun, et al. Transient Characteristics of Inductive Motor in Limited Capacity Power System[J]. Journal of North China Electric Power University2016,

[3] M.A.Tankari,M.B.Camara,B,Dakyo,et al.Use of Ultraca-pacitors and Batteries For Efficient Energy Management in Wind-Dies-el Hybrid system[J].IEEE Trans.Sustainable Energy,2013,4(2):414-424.

[4] WEI Gang, CHEN Senhuan, CAI Yang, et al. Compensation for three-phase unbalanced load based on instantaneous reacti-ve power theory[J]. Electric Power Automation Equipment, 2010, 30(2): 59-64.

[5] HOU Shiying, PAN Chong, LV Houyu, et al. Comparative Study of Three phase Four wire System Instantaneous Power Theory [J]. High Voltage Engineering, 2007, 33(5): 114-118.

[6] LIU Tao, DAI Hanyang, SONG Xinli, et al. A Typical Wind Power Generation Set Model of Suitable for Full Dynamic Simulation of Power Grid[J]. Power System Technology, 2015, 39(3): 609-614.

[7] Zhang Jianhua, Yu Lei, Liu Nian, et al. Capacity Configuration Optimization for Island Microgrid with Wind/Photovoltaic/Diesel/Storae and Seawater Desalination Load [J].TRANSACTIONS OF CHINA ELECTROTECHNICAL SOCIETY, 2014, 29(2): 102-113. 